\section{Detection of Interleaved OFDMA Uplink Signals in the Presence of Residual Frequency Offset Using the SAGE Algorithm}

\author{
Jong-Ho Lee and Seong-Cheol Kim, Member, IEEE
}

\begin{abstract}
In this paper, we propose an iterative detection scheme in the presence of residual frequency offsets (RFOs) using the spacealternating generalized expectation-maximization algorithm for an orthogonal-frequency-division-multiple-access uplink system. In the proposed scheme, the expectation step intends to remove intercarrier interference (ICI) due to the RFOs of simultaneous users in the received signals. Then, the maximization step is utilized to estimate the required parameters for each user (i.e., RFO, data symbols, and channel state information) employing the ICI-cancelled signals. Simulation results show that the proposed scheme achieves almost ideal bit-error-rate performance as long as the magnitude of the normalized RFO value is within 0.2 .
\end{abstract}

Index Terms-Orthogonal frequency-division multiple access (OFDMA), residual frequency offset (RFO), space-alternating generalized expectation-maximization (SAGE).

\section{INTRODUCTION}

Orthogonal frequency-division multiple access (OFDMA) is a multiplexing scheme where each user employs a different set of orthogonal subcarriers to transmit data symbols simultaneously. The different orthogonal subcarrier sets for different users in the OFDMA system make it possible to avoid multiple access interference (MAI). Therefore, it has become an attractive solution for various wireless communication systems [1]-[3].

It is known that, in the OFDMA uplink system, carrier-frequencysynchronization issues become even more crucial [3]. In case of imperfect carrier frequency synchronization, intercarrier interference (ICI) is generated due to the loss of subcarrier orthogonality, which induces both cochannel and interchannel interferences. Moreover, carrier frequency synchronization in the OFDMA uplink is more difficult since the frequency recovery for one user may result in the misalignment of other synchronized users.

Timing and frequency synchronization schemes for OFDMA uplink system have been studied in [2]-[7]. Through downlink control channels, the estimated offsets in the base station (BS) were returned to each user's terminal, where timing and frequency offsets are actually adjusted. In [2], user separation is performed by the grouping of adjacent subcarriers, which divides the available spectrum into several subbands. Then, each user is allocated in one of the subbands, and the BS obtains the user-separated signals in the time domain through the subband filters. Then, the synchronization scheme in [2] estimates the timing and frequency offsets by repetition of the cyclic prefix (CP) [8]. The CP-based estimator does not need any reference signals and

Manuscript received May 26, 2005; revised November 20, 2005, March 6, 2006, July 9, 2006, and July 10, 2006. This work was supported in part by the University IT Research Center Project and in part by the Brain Korea 21 Project. The review of this paper was coordinated by Prof. G. Saulnier.

J.-H. Lee was with the Institute of New Media and Communications, School of Electrical Engineering and Computer Science, Seoul National University, Seoul 151-742, Korea. He is now with the Next Generation System Team, Samsung Electronics Company, Ltd., Korea (e-mail: jh0512.lee@samsung.com).

S.-C. Kim is with the Institute of New Media and Communications, School of Electrical Engineering and Computer Science, Seoul National University, Seoul 151-742, Korea (e-mail: sckim@maxwell.snu.ac.kr).

Digital Object Identifier 10.1109/TVT.2007.895574 is relatively simple. However, in the subband-based OFDMA uplink, deep fading due to the frequency-selective characteristic in multipathfading channels may lie in several adjacent subcarriers, so that full channel diversity is not guaranteed.

In order to utilize channel diversity for all users, the interleaved subcarrier set can be assigned to each user [3]-[6]. Then, the userseparated signals are available only in the frequency domain. In particular, for the interleaved OFDMA uplink, Morelli [3] proposed a time and frequency synchronization scheme that considers the correlation properties of the frequency-domain received signals. It is known that the synchronization scheme in [3] achieves sufficient accuracy of the timing and frequency-offset estimates and is applicable to the generalized OFDMA uplink, while it needs several preamble symbols.

In this paper, we consider the situation where carrier frequency synchronization in the preamble is imperfect, so that residual frequency offsets (RFOs) are induced in the interleaved OFDMA uplink. The RFOs of all simultaneous users cause MAI and significantly degrade the transmission performance [9]-[11]. In particular, for the RFO estimation in single-user OFDM, Lui and Tureli [10], and Attallah [11] employ virtual subcarriers that are not actually modulated, considering the orthogonality between subcarriers. However, it is seen that these schemes are not directly applicable to multiuser OFDM systems because the virtual subcarriers suffer from the different RFOs of different active users in the given system. In [4], the inherent signal structure of an equally spaced subcarrier set is utilized to estimate the RFOs in the interleaved OFDMA uplink, while it requires a MUltiple SIgnal Classification-like search. Moreover, this scheme is available when the number of active users is less than the total number of interleaved subcarrier sets or extra $\mathrm{CP}$ is appended to guarantee reliable performance. The RFO correction scheme is also proposed in [5] based on the least square (LS) and minimum-mean-square-error criteria, assuming that the RFOs are known to the BS. Here, we focus on a joint RFO, channel-estimation, and data-detection scheme, which is applicable to any subcarrier assignment strategy.

The proposed RFO compensation scheme is based on the results of [12] and employs the space-alternating generalized expectation-maximization (SAGE) algorithm [13], [14]. The expectation step in the proposed scheme intends to cancel the ICI due to the RFOs of all users in the received signals. Then, the maximization step is employed to estimate the required parameters for each user [i.e., RFO, channel state information (CSI), and data symbols]. The rest of this paper is organized as follows. Section II describes the OFDMA uplink system model considered in this paper. In Section III, we propose an iterative RFO compensation scheme based on the SAGE algorithm. Section IV shows the performance of the proposed algorithm that is accessed by computer simulations, and Section $\mathrm{V}$ presents the concluding remarks.

\section{System ModeL}

Let $N$ and $K$ be the number of subcarriers and the number of users, respectively. The number of subcarriers that is assigned to each user can be given as $M=N / K$. Then, the data symbol vector of the $k$ th user $\mathbf{s}_{k}=\left[s_{k}(1) s_{k}(2) \cdots s_{k}(M)\right]^{T}$ modulates the subcarriers that are assigned to the $k$ th user, which can be indexed by the subcarrier index vector $\mathbf{c}_{k}=\left[c_{k}(1) c_{k}(2) \cdots c_{k}(M)\right]^{T}$, with $1 \leq c_{k}(m) \leq N$. Note that we do not regulate any specific manner of the interleaved subcarrier assignment.

At first, we assume that the timing synchronization is perfect, while carrier frequency synchronization is inaccurate in the preamble. Then, 
the time-domain received signal vector $\mathbf{y}=[y(1) y(2) \cdots y(N)]^{T}$ with frequency synchronization errors can be expressed as

$$
\mathbf{y}=\sum_{k=1}^{K} \boldsymbol{\Gamma}\left(v_{k}\right) \mathbf{F} \mathbf{S}_{k} \mathbf{H}_{k}+\mathbf{w}
$$

where the normalized RFO $v_{k}$ for the $k$ th user is presented in the diagonal matrix $\boldsymbol{\Gamma}\left(v_{k}\right)$ that is given by

$$
\boldsymbol{\Gamma}\left(v_{k}\right)=\operatorname{diag}\left\{e^{j \frac{2 \pi v_{k}}{N}(n-1)}, 1 \leq n \leq N\right\}
$$

and the inverse discrete Fourier transform matrix is given by

$$
[\mathbf{F}]_{n, p}=\frac{1}{\sqrt{N}} e^{j \frac{2 \pi(p-1)}{N}(n-1)}, \quad 1 \leq n ; \quad p \leq N .
$$

The diagonal data symbol matrix $\mathbf{S}_{k}$ in (1) can be defined as

$$
\left[\mathbf{S}_{k}\right]_{p, p}= \begin{cases}s_{k}(m), & \text { if } p=c_{k}(m) \\ 0, & \text { otherwise. }\end{cases}
$$

The channel frequency response for the $k$ th user can be given as $\mathbf{H}_{k}=\mathbf{D h}_{k}$, where $\mathbf{h}_{k}=\left[\begin{array}{llll}h_{k}(1) & h_{k}(2) & \cdots & h_{k}(L)\end{array}\right]^{T}$ denotes the channel impulse response (CIR) with $L$ multipaths for the $k$ th user and $[\mathbf{D}]_{p, l}=e^{-j(2 \pi(p-1) / N)(l-1)}, l=1,2, \ldots, L$. Moreover, $\mathbf{w}=$ $[w(1) w(2) \cdots w(N)]^{T}$ in (1) presents the additive white Gaussian noise vector with the covariance matrix of $\sigma^{2} \mathbf{I}$.

Here, we assume that all users are ideally time synchronized. Note that ideal timing synchronization for all users is not possible in practical situations. In order to avoid intersymbol interference due to timing-synchronization errors, it is assumed that the length of the guard interval is selected to cope with the maximum channel delay spread and the maximum timing error [4]. Then, the timing errors induce only the phase rotation [15].

\section{Detection OF OFDMA UpLink SignAL}

\section{A. Proposed Scheme}

The frequency-domain received signal vector can be written as

$$
\begin{aligned}
\mathbf{Y} & =\mathbf{F}^{H} \mathbf{y} \\
& =\sum_{k=1}^{K} \varepsilon_{k} \mathbf{S}_{k} \mathbf{D} \mathbf{h}_{k}+\sum_{k=1}^{K} \mathbf{R}_{k} \mathbf{S}_{k} \mathbf{D} \mathbf{h}_{k}+\mathbf{v}
\end{aligned}
$$

where $(.)^{H}$ denotes the conjugated transpose, and $\mathbf{v}=\mathbf{F}^{H} \mathbf{w}$. In (5), $\varepsilon_{k}$ is a common RFO error [12] for the $k$ th user, which is defined as

$$
\begin{aligned}
\varepsilon_{k} & =\frac{1}{N} \sum_{n=0}^{N-1} e^{j \frac{2 \pi v_{k}}{N} n} \\
& =\frac{1}{N} \frac{1-e^{j 2 \pi v_{k}}}{1-e^{\frac{j 2 \pi v_{k}}{N}}}
\end{aligned}
$$

Assuming that $v_{k}$ has a sufficiently small value and $\varepsilon_{k}$ is given, Taylor's series expansion $\left(e^{j(2 \pi v / N)} \approx 1+j(2 \pi v / N)\right)$ is applied to the denominator in (6). Then, we can obtain $v_{k}$, which is approximately given as

$$
v_{k} \approx \frac{\operatorname{Im}\left\{\varepsilon_{k}\right\}}{\pi\left|\varepsilon_{k}\right|^{2}}
$$

Moreover, the $N \times N$ matrix $\mathbf{R}_{k}$ in (5) induces the ICI due to RFO $v_{k}$, which is defined as

$$
\mathbf{R}_{k}=\mathbf{F}^{H} \boldsymbol{\Gamma}\left(v_{k}\right) \mathbf{F}-\varepsilon_{k} \mathbf{I}_{N}
$$

where $\mathbf{I}_{N}$ denotes an $N \times N$ identity matrix. Then, the diagonal entries of $\mathbf{R}_{k}$ are equal to zero, and the off-diagonal entries are given by

$$
\begin{aligned}
{\left[\mathbf{R}_{k}\right]_{p, q} } & =\frac{1}{N} \sum_{n=0}^{N-1} e^{j \frac{2 \pi\left(q-p+v_{k}\right)}{N} n} \\
& =\frac{1}{N} \frac{1-e^{j 2 \pi\left(q-p+v_{k}\right)}}{1-e^{j \frac{2 \pi\left(q-p+v_{k}\right)}{N}}}
\end{aligned}
$$

where $1 \leq p$ and $q \leq N$.

In the proposed scheme based on the SAGE algorithm, the frequency-domain received signal $\mathbf{Y}$ in (5) is divided into the desired signal

$$
\mathbf{Y}_{D}=\sum_{k=1}^{K} \varepsilon_{k} \mathbf{S}_{k} \mathbf{D h}_{k}+\beta_{D} \mathbf{v}
$$

and the interference signal

$$
\mathbf{Y}_{I}=\sum_{k=1}^{K} \mathbf{R}_{k} \mathbf{S}_{k} \mathbf{D h}_{k}+\beta_{I} \mathbf{v}
$$

where the nonnegative parameters $\beta_{D}$ and $\beta_{I}$ satisfy $\beta_{D}^{2}+\beta_{I}^{2}=1$ [16], [17]. Then, we try to estimate $\varepsilon_{k}, \mathbf{s}_{k}$, and $\mathbf{h}_{k}$ for all users using the desired signal $\mathbf{Y}_{D}$ in (10) [12]. Note that, as shown in (10) and (11), the two kinds of RFO effects in the frequency-domain received signals are the common RFO error and the ICI [12]. In particular, the loss of the desired signal power $10 \log _{10}\left(\left|\varepsilon_{k}\right|^{2}\right)$ is more than $0.5 \mathrm{~dB}$ when the normalized RFO value is above 0.2 .

In the expectation step at the $i$ th iteration, the following expectations are evaluated:

$$
\begin{aligned}
\mathbf{Y}_{D}^{(i)}= & E\left[\mathbf{Y}_{D} \mid \varepsilon^{(i)}, \mathbf{s}^{(i)}, \mathbf{h}^{(i)}\right] \\
= & \sum_{k=1}^{K} \varepsilon_{k}^{(i)} \mathbf{S}_{k}^{(i)} \mathbf{D} \mathbf{h}_{k}^{(i)} \\
& +\beta_{D}^{2}\left(\mathbf{Y}-\sum_{k=1}^{K} \varepsilon_{k}^{(i)} \mathbf{S}_{k}^{(i)} \mathbf{D} \mathbf{h}_{k}^{(i)}-\sum_{k=1}^{K} \mathbf{R}_{k}^{(i)} \mathbf{S}_{k}^{(i)} \mathbf{D} \mathbf{h}_{k}^{(i)}\right) \\
\mathbf{Y}_{I}^{(i)}= & E\left[\mathbf{Y}_{I} \mid \varepsilon^{(i)}, \mathbf{s}^{(i)}, \mathbf{h}^{(i)}\right] \\
= & \sum_{k=1}^{K} \mathbf{R}_{k}^{(i)} \mathbf{S}_{k}^{(i)} \mathbf{D} \mathbf{h}_{k}^{(i)} \\
& +\beta_{I}^{2}\left(\mathbf{Y}-\sum_{k=1}^{K} \varepsilon_{k}^{(i)} \mathbf{S}_{k}^{(i)} \mathbf{D} \mathbf{h}_{k}^{(i)}-\sum_{k=1}^{K} \mathbf{R}_{k}^{(i)} \mathbf{S}_{k}^{(i)} \mathbf{D} \mathbf{h}_{k}^{(i)}\right)
\end{aligned}
$$

where $\varepsilon^{(i)}, \mathbf{s}^{(i)}$, and $\mathbf{h}^{(i)}$ denote the $i$ th estimates of $\varepsilon=$ $\left[\begin{array}{llll}\varepsilon_{1} & \varepsilon_{2} & \cdots & \varepsilon_{K}\end{array}\right]^{T}, \mathbf{s}=\left[\begin{array}{llll}\mathbf{s}_{1}^{T} & \mathbf{s}_{2}^{T} & \cdots & \mathbf{s}_{K}^{T}\end{array}\right]^{T}$, and $\mathbf{h}=\left[\begin{array}{llll}\mathbf{h}_{1}^{T} & \mathbf{h}_{2}^{T} & \cdots & \mathbf{h}_{K}^{T}\end{array}\right]^{T}$, respectively. Moreover, in (12) and (13), $\mathbf{R}_{k}^{(i)}=\mathbf{F}^{H} \boldsymbol{\Gamma}_{v^{(i)}} \mathbf{F}-\varepsilon_{k}^{(i)} \mathbf{I}_{N}$, where $v_{k}^{(i)} \approx\left(\operatorname{Im}\left\{\varepsilon_{k}^{(i)}\right\} / \pi\left|\varepsilon_{k}^{(i)}\right|^{2}\right)$. It is seen that the expectation step 
of the proposed algorithm is equivalent to the interference cancellation in the received signals [12].

The maximization step of the $i$ th iteration using the desired signal $\mathbf{Y}_{D}^{(i)}$ in (12) evaluates the following equations:

$$
\begin{aligned}
& \varepsilon^{(i+1)}=\underset{\varepsilon}{\arg \max \Lambda}\left(\tilde{\varepsilon}, \mathbf{s}^{(i)}, \mathbf{h}^{(i)} \mid \mathbf{Y}_{D}^{(i)}\right) \\
& v_{k}^{(i+1)} \approx \frac{\operatorname{Im}\left\{\varepsilon_{k}^{(i+1)}\right\}}{\pi\left|\varepsilon_{k}^{(i+1)}\right|^{2}}, k=1,2, \ldots, K \\
& \mathbf{s}^{(i+1)}=\underset{\mathbf{s}}{\arg \max \Lambda}\left(\varepsilon^{(i+1)}, \tilde{\mathbf{s}}, \mathbf{h}^{(i+1)} \mid \mathbf{Y}_{D}^{(i)}\right) \\
& \mathbf{h}^{(i+1)}=\underset{\mathbf{h}}{\arg \max \Lambda}\left(\varepsilon^{(i+1)}, \mathbf{s}^{(i+1)}, \tilde{\mathbf{h}} \mid \mathbf{Y}_{D}^{(i)}\right)
\end{aligned}
$$

where the log-likelihood function $\Lambda\left(\varepsilon, \mathbf{s}, \mathbf{h} \mid \mathbf{z}_{D}\right)$, assuming constant envelope modulation schemes, can be given as

$$
\begin{aligned}
& \Lambda\left(\varepsilon, \mathbf{s}, \mathbf{h} \mid \mathbf{Y}_{D}\right) \\
& \quad=\frac{1}{\beta_{D}^{2} \sigma_{v}^{2}}\left[2 \operatorname{Re}\left\{\mathbf{Y}_{D}^{H} \sum_{k=1}^{K} \varepsilon_{k} \mathbf{S}_{k} \mathbf{D} \mathbf{h}_{k}\right\}-\sum_{k=1}^{K} M\left|\varepsilon_{k}\right|^{2} \mathbf{h}_{k}^{H} \mathbf{h}_{k}\right] .
\end{aligned}
$$

Note that $\left(\mathbf{S}_{k} \mathbf{D}\right)^{H} \mathbf{S}_{k} \mathbf{D}=M \cdot \mathbf{I}_{L}$ and $\mathbf{I}_{L}$ is an $L \times L$ identity matrix. Then, the solution of (14) can be evaluated as

$$
\varepsilon_{k}^{(i+1)}=\frac{1}{M\left|\mathbf{h}_{k}^{(i)}\right|^{2}}\left(\mathbf{S}_{k}^{(i)} \mathbf{D} \mathbf{h}_{k}^{(i)}\right)^{H} \mathbf{Y}_{D}^{(i)}
$$

Moreover, applying the LS solution to (17) yields

$$
\mathbf{h}_{k}^{(i+1)}=\frac{\left(\varepsilon_{k}^{(i+1)}\right)^{*}}{M\left|\varepsilon_{k}^{(i+1)}\right|^{2}}\left(\mathbf{S}_{k}^{(i+1)} \mathbf{D}\right)^{H} \mathbf{Y}_{D}^{(i)}
$$

\section{B. Initialization}

The initial CSI estimate $\mathbf{h}^{(0)}$ can be obtained by the channel estimate in the previous OFDM symbol, assuming that the channel variation between adjacent OFDM symbols is negligible. In order to obtain the initial estimates of $\varepsilon^{(0)}$ and $\mathbf{s}^{(0)}$, it is assumed that each user has pilot subcarriers. Note that the initial estimates of $\varepsilon^{(0)}$ and $\mathbf{s}^{(0)}$ should be evaluated by the received signal including ICI because the interference cancellation cannot be operated without the information of $\mathbf{s}$.

In order to obtain $\varepsilon_{k}^{(0)}$, we use pilot subcarriers that are embedded in the orthogonal subcarrier set of each user given as

$$
\varepsilon_{k}^{(0)}=\frac{1}{\left|\mathbf{S}_{k}^{\text {pilot }} \mathbf{D}_{k}^{\text {pilot }} \mathbf{h}_{k}^{(0)}\right|^{2}}\left(\mathbf{S}_{k}^{\text {pilot }} \mathbf{D}_{k}^{\text {pilot }} \mathbf{h}_{k}^{(0)}\right)^{H} \mathbf{Y}_{k}^{\text {pilot }}
$$

where $\mathbf{Y}_{k}^{\text {pilot }}, \mathbf{S}_{k}^{\text {pilot }}$, and $\mathbf{D}_{k}^{\text {pilot }}$ denote the received signal vector at the pilot subcarriers, the diagonal pilot symbol matrix, and the DFT matrix that is composed of only pilot subcarriers for the $k$ th user, respectively. Moreover, given $\varepsilon_{k}^{(0)}$, we can obtain $v_{k}^{(0)}$, as

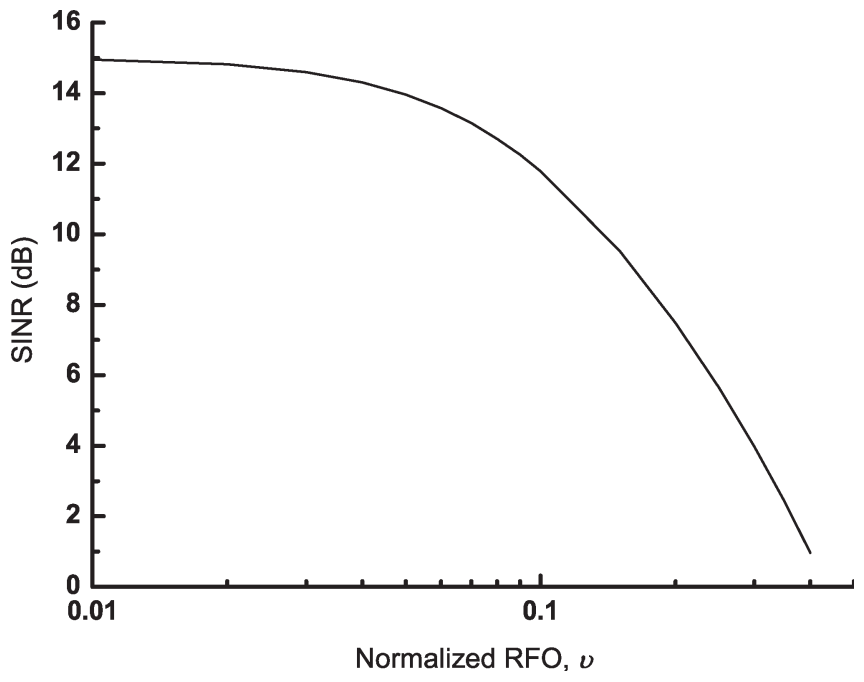

Fig. 1. Average SINR with different RFO values $(\mathrm{SNR}=15 \mathrm{~dB})$.

shown in (7). For $\mathbf{s}^{(0)}$, we evaluate the following equation using $\varepsilon^{(0)}$ and $\mathbf{h}^{(0)}$.

$$
\mathbf{s}^{(0)}=\underset{\mathbf{s}}{\arg \max } \Lambda\left(\varepsilon^{(0)}, \mathbf{s}, \mathbf{h}^{(0)} \mid \mathbf{Y}\right)
$$

\section{Simulation Result}

A summary of the system parameters for our simulation is provided here.

- The total number of subcarriers is $N=256$.

- The power delay profile of CIR is given as $E\left|h_{k}(l)\right|^{2} \propto$ $e^{-(l-1) / 4}, 1 \leq l \leq 6$, and each multipath varies according to Rayleigh distribution [20].

- The total number of users is $K=8$.

- Each user has uniformly spaced subcarriers from each other, which indicates that the subcarrier index for the $k$ th user is given by $c_{k}(m)=k+(m-1) K, 1 \leq m \leq M$.

- Three pilot subcarriers are embedded in the subcarrier set that is assigned to each user.

- One OFDMA packet is composed of 50 OFDM symbols, and the first symbol of each packet is set to be a preamble symbol.

- The subcarriers are modulated by quadrature phase-shift keying.

- For time-varying channels (TVCs), the carrier frequency and vehicle speed are set to be $5 \mathrm{GHz}$ and $60 \mathrm{~km} / \mathrm{h}$, respectively.

We considered two channel conditions, namely: 1) the static channel (SC) and 2) the TVC. The SC indicates that the channel is constant over a packet and the CSI is available at the receiver. Then, there is no need to include the process for CSI in (17). For the TVC, which presents that the channel variation should be tracked at each OFDM symbol, the proposed algorithm should include the CSI process in (17). It is assumed that the RFOs are constant in each packet and the process for RFO estimator in (14) and (15) is performed only at the first OFDM data symbol after the preamble symbols. The final estimate of the RFO is utilized to detect the following OFDM data symbols in the packet. Moreover, the initial channel estimate at the first data symbol is obtained at the synchronization stage using the preamble symbols.

For illustration purposes, we set the RFOs of all users to the same value. At first, we analyze performance degradation due to RFOs in 


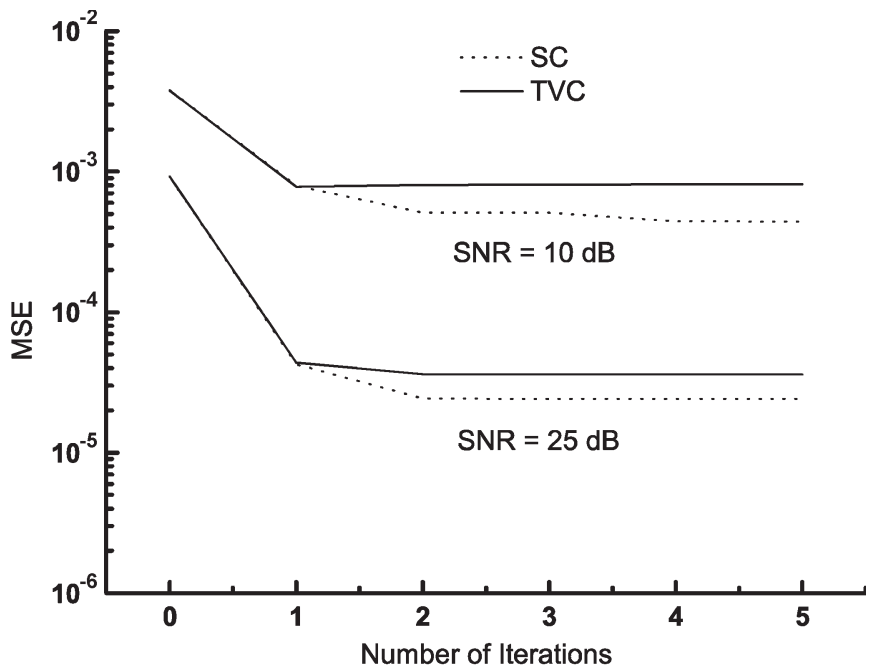

(a)

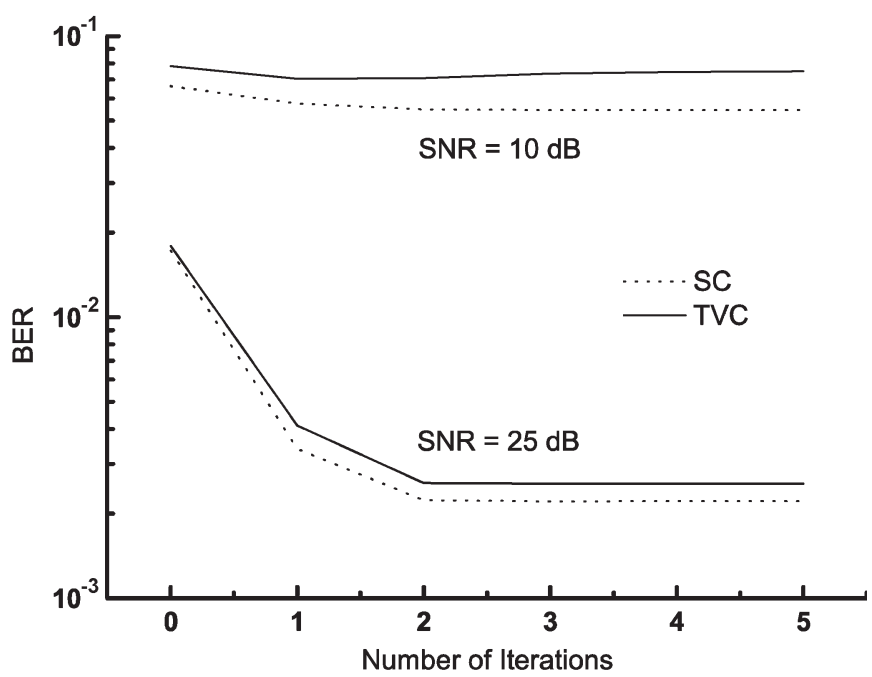

(b)

Fig. 2. Convergence properties of the proposed algorithm $(v=0.1)$. (a) MSE performance of RFO estimator. (b) BER performance.

terms of the signal-to-interference-and-noise ratio (SINR). From (5), the ICI at the $p$ th subcarrier that is assigned to the $k$ th user can be given as $\sum_{m=1}^{K} \sum_{q=1}^{M}\left|\left[\mathbf{R}_{m}\right]_{c_{k}(p), c_{m}(q)}\right|^{2}$. Then, the average SINR is defined as

$$
\operatorname{SINR}=\frac{\frac{1}{K} \sum_{k=1}^{K}\left|\varepsilon_{k}\right|^{2}}{\frac{1}{N} \sum_{k=1}^{K} \sum_{p=1}^{M} \sum_{m=1}^{K} \sum_{q=1}^{M}\left|\left[\mathbf{R}_{m}\right]_{c_{k}(p), c_{m}(q)}\right|^{2}+\sigma^{2}} .
$$

Fig. 1 shows the average SINR as a function of RFO. It is observed that the SINR rapidly decreases and the SINR loss is more than $5 \mathrm{~dB}$ as the normalized RFO value increases above 0.1 .

Fig. 2 shows the convergence properties of the proposed iterative scheme. It is seen that the mean square error (MSE) of the RFO estimator and the bit error rate (BER) performance are almost converged after two iterations. Therefore, we fix the number of iterations to two for the proposed scheme in the following results.

In Figs. 3 and 4, we compare the MSE performance of the proposed algorithm with the Cramer-Rao bounds (CRBs) for RFO and channel

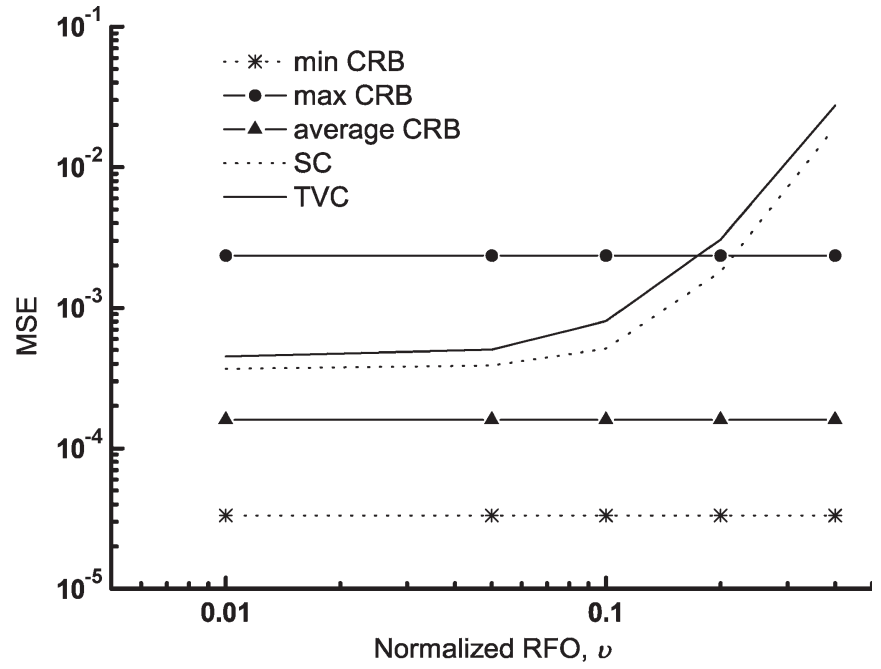

(a)

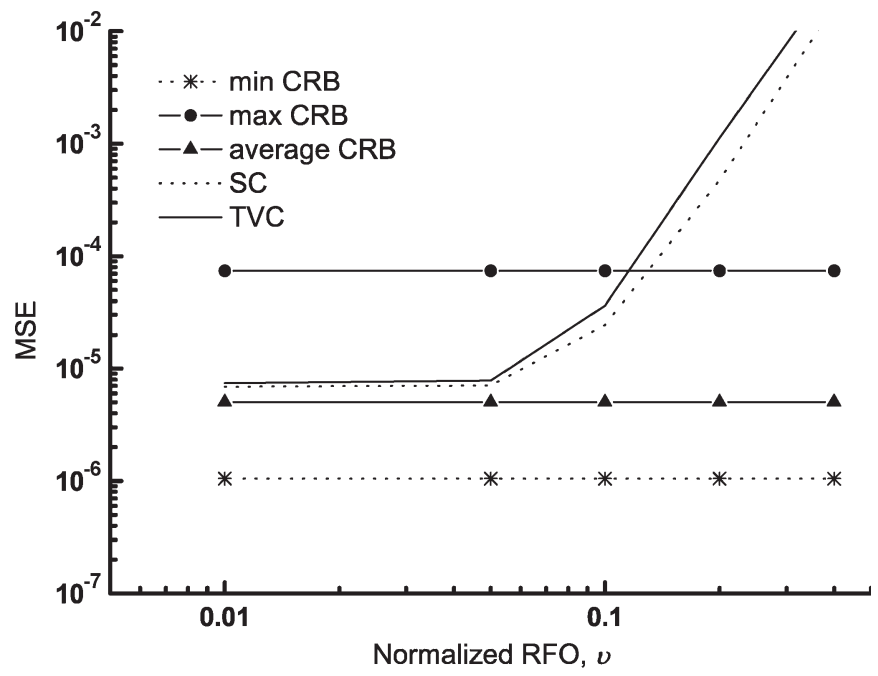

(b)

Fig. 3. Comparison of the MSE performance for an RFO estimator with different values of RFO $v$. (a) $\mathrm{SNR}=10 \mathrm{~dB}$. (b) $\mathrm{SNR}=25 \mathrm{~dB}$.

estimation according to the different values of RFO. In Fig. 3, the minimum, maximum, and average CRBs that are given in [7] are evaluated by $10^{5}$ simulation runs. It is seen that the RFO and channel estimators of the proposed algorithm show comparable MSE performance with the CRBs. Note that the MSE performance of the proposed scheme in Figs. 3 and 4 is gradually degraded as the normalized RFO value increases because of the desired signal power loss and the approximation using Taylor's series expansion in (7).

Fig. 5 shows the BER performance of the proposed scheme as a function of the normalized RFO, compared with the ideal performance in perfect frequency synchronization and that without RFO compensation for known CSI. As shown in Fig. 5, the proposed scheme remarkably compensates performance degradation due to the RFOs. In Fig. 5(a), it is seen that the performance without RFO compensation for known CSI is better than that of the proposed scheme in TVC since the performance degradation due to the channel estimation error is more dominant than that due to RFO at low signal-to-noise ratio (SNR) with small RFO value.

In order to investigate the performance of the proposed algorithm with the different RFO values of different users, we only fix user 1's RFO $v_{1}$, and the others $\left(v_{k}, k=2, \ldots, K\right)$ were randomly chosen 


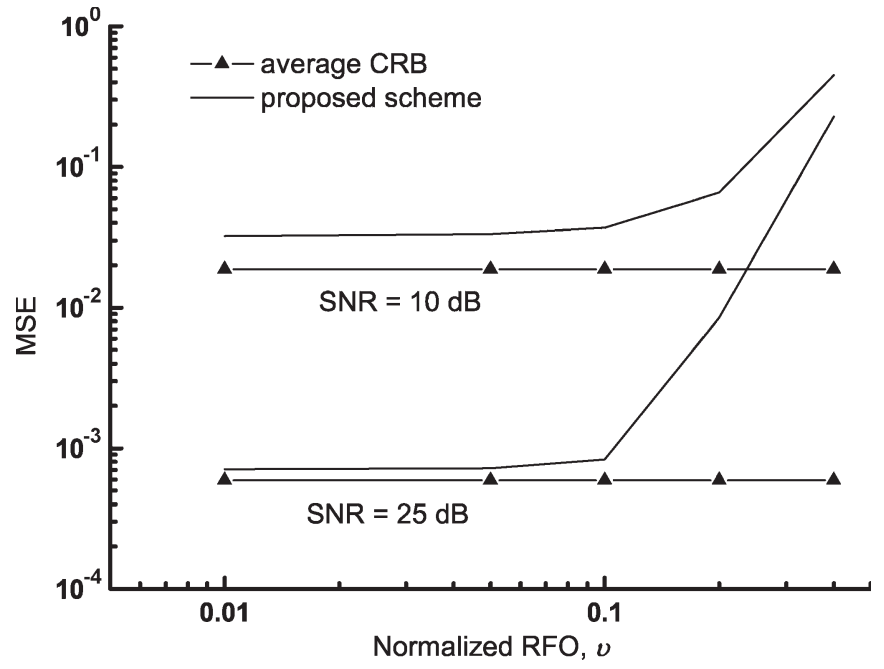

Fig. 4. Comparison of the MSE performance for a channel estimator with different values of RFO $v$.

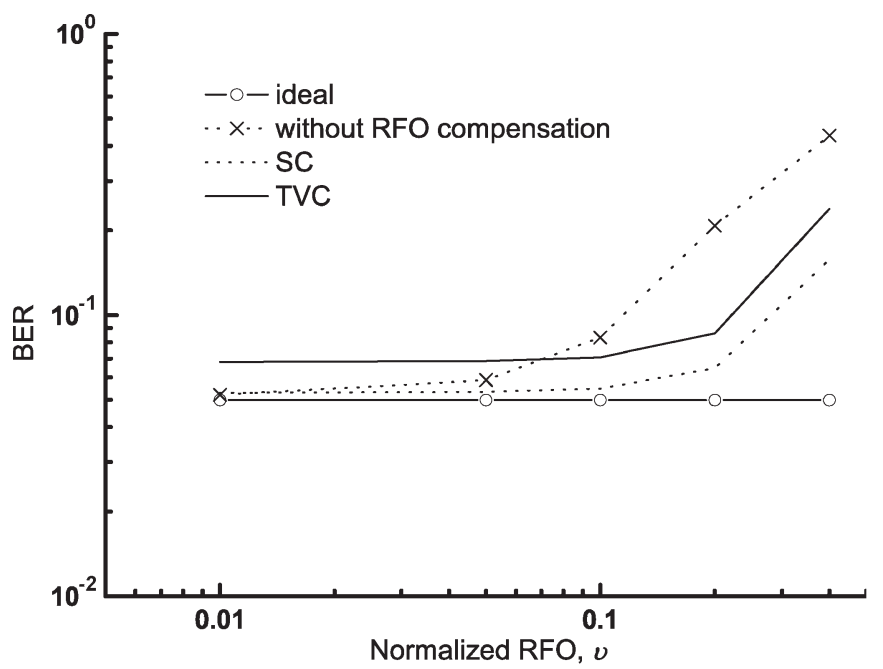

(a)

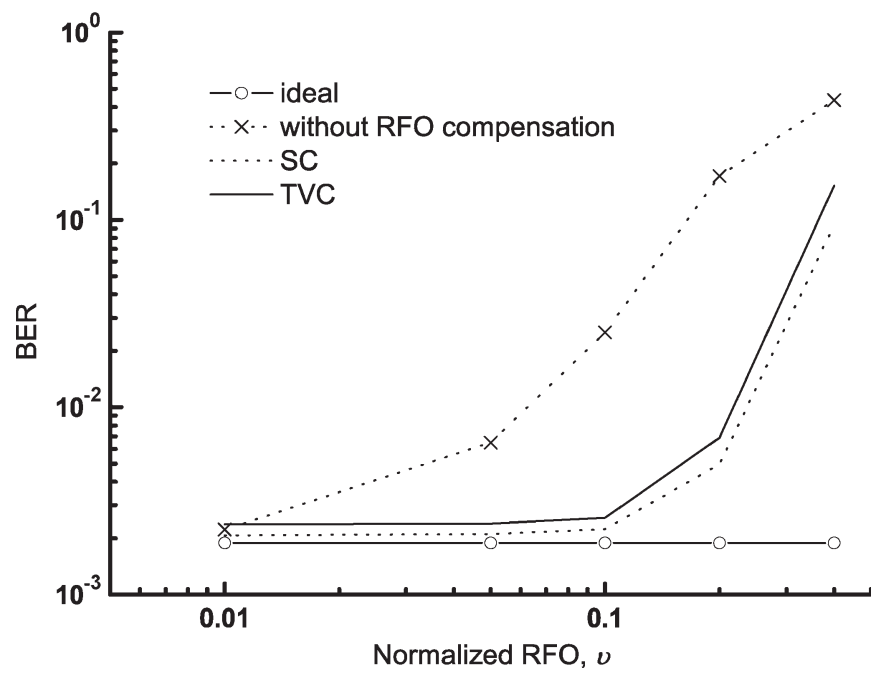

(b)

Fig. 5. Comparison of the BER performance according to different values of $\mathrm{RFO} v$. (a) $\mathrm{SNR}=10 \mathrm{~dB}$. (b) $\mathrm{SNR}=25 \mathrm{~dB}$.

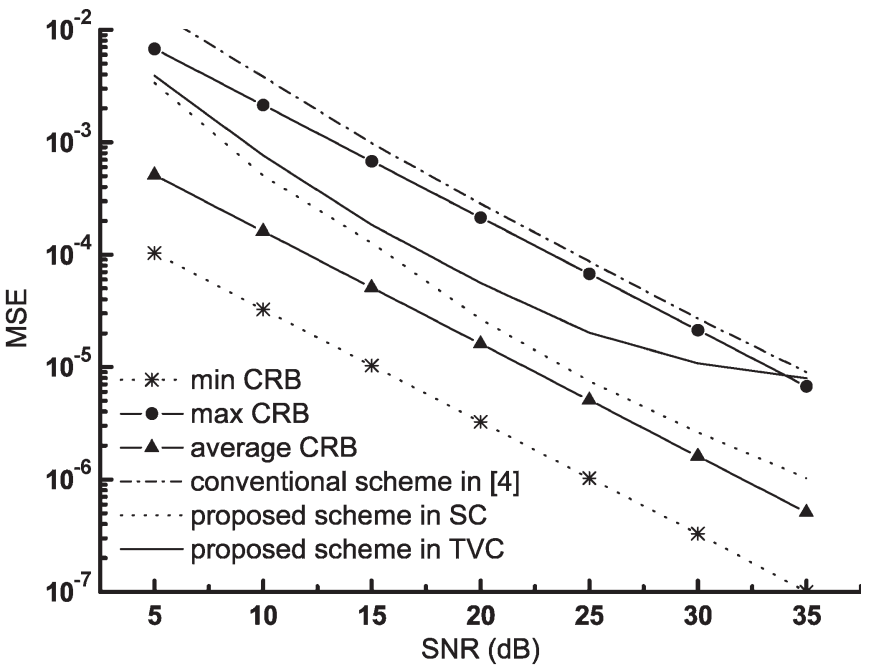

(a)

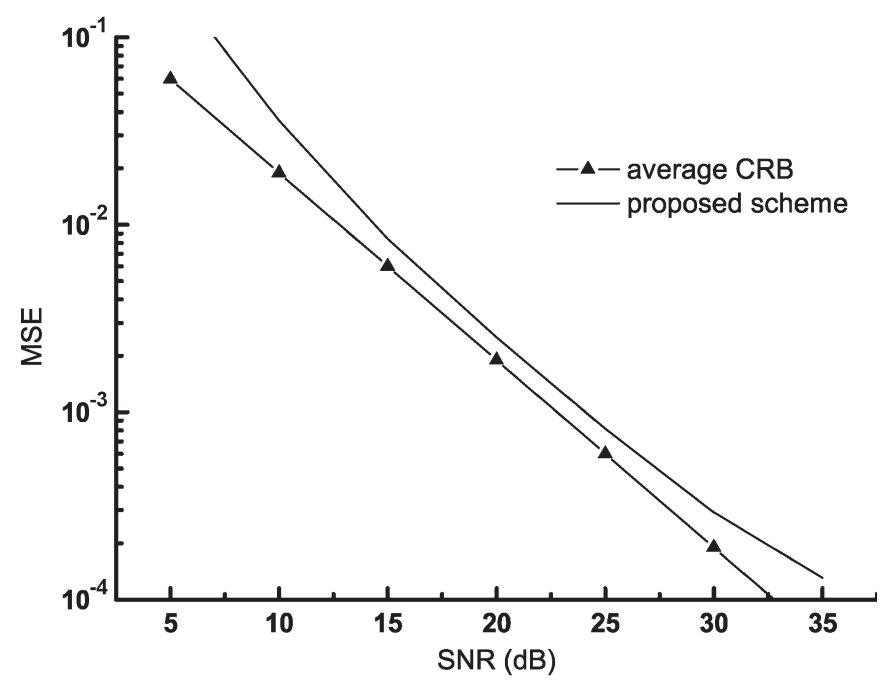

(b)

Fig. 6. MSE performance versus SNR compared with CRBs $\left(v_{1}=0.1\right)$. (a) MSE performance of RFO estimation. (b) MSE performance of CIR estimation.

between -0.2 and 0.2 in the following simulation results. Fig. 6 shows the MSE performance versus SNR for the RFO and CIR estimators of the proposed scheme. In Fig. 6(a), the performance of the conventional scheme in [4] is evaluated, assuming that only six users are active in the system at $K=8$. It can be observed that the MSE for RFO estimation in the proposed scheme lies between the maximum and minimum CRBs in all ranges of SNR. In SCs, the MSE of the proposed RFO estimator is close to the average CRB, while it shows a floor at high SNR in TVCs. Moreover, the proposed RFO estimator outperforms the conventional scheme [4] in all ranges of SNR. In Fig. 6(b), the MSE of the CIR estimator also approaches the CRB [7] as the SNR increases.

The BER performance of the proposed scheme is presented as a function of SNR with $v_{1}=0.2$ in Fig. 7. The BER performance of [18] is also evaluated and compared with that of the proposed scheme. The conventional scheme in [18] is based on interference cancellation and RFO correction using circular convolution. In order to detect data symbols, [18] employs three circular convolutions with one iteration, while the proposed scheme performs two expectation steps with two iterations. Note that both the circular convolution in [18] and the expectation step in the proposed scheme need the computational 


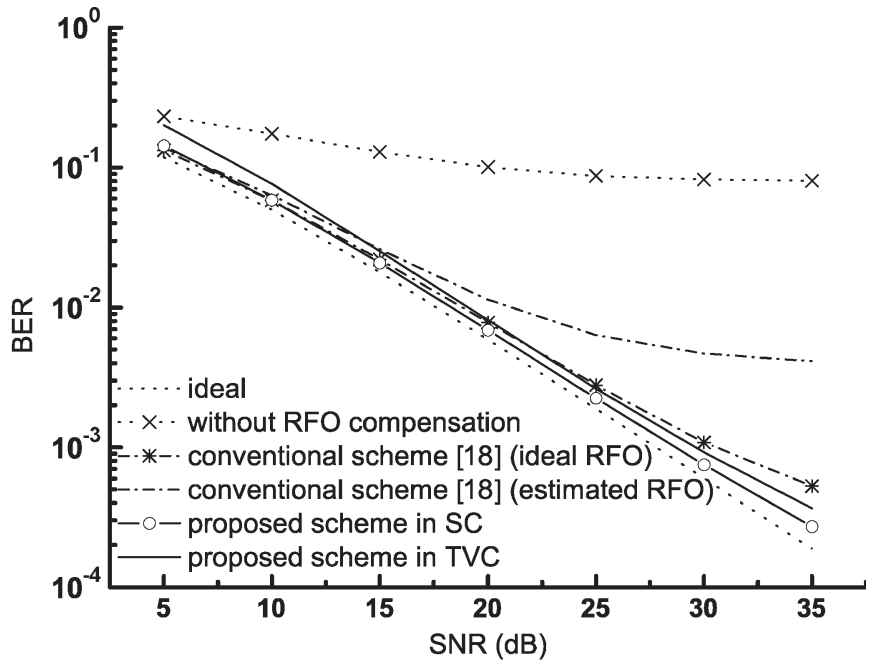

Fig. 7. Comparison of BER performance versus SNR $\left(v_{1}=0.2\right)$.

complexity of $O\left(N^{2}\right)$. However, in order to reduce complexity, some elements in the operation of circular convolution are set to zero in [18], assuming that the normalized RFO is very small. Moreover, it employs the frequency-offset estimator for a single-user OFDM system [19] to obtain the RFO estimates for all users. As the number of users and the RFO value of each user increase, the RFO estimates become quite inaccurate due to the MAI, so that the RFO correction in [18] may not be effective. Note that, in our simulations, the performance of the conventional RFO correction scheme is evaluated with one iteration and known CSI, as given in [18].

As shown in Fig. 7, the BER performance of [18] with ideal RFO values is comparable to that of the proposed scheme, while an error floor exists at high SNR in the conventional scheme with estimated RFO values using [19]. Moreover, it is obvious that the proposed scheme outperforms the conventional RFO correction scheme in all ranges of SNR and significantly compensates the degraded performance due to the RFOs of simultaneous users.

\section{CONCLUSION}

In this paper, we proposed an iterative detection scheme using the SAGE algorithm in the presence of RFOs for an interleaved OFDMA uplink system. The expectation step in the proposed scheme extracts the desired terms from the received signals. Then, the maximization step is utilized to estimate the RFO, data symbols, and CSI using the desired signals for each user. Simulation results show that the proposed scheme needs two iterations to obtain converged estimates and significantly compensates the performance degradation due to the RFOs of simultaneous users. Moreover, it outperforms the conventional schemes and also achieves almost ideal BER performance, while the normalized RFO value is within 0.2 .

\section{ACKNOWLEDGMENT}

The authors would like to thank the paper editor and the reviewers for their valuable comments and suggestions.

\section{REFERENCES}

[1] P. Xia, S. Zhou, and G. B. Giannakis, "Bandwidth- and power-efficient multicarrier multiple access," IEEE Trans. Commun., vol. 51, no. 11, pp. 1828-1837, Nov. 2003.

[2] J. J. van de Beek, P. O. Borjesson, M. L. Boucheret, D. Landstram, J. M. Arenas, P. Odling, C. Ostberg, M. Wahlqvist, and S. K. Wilson,
"A time and frequency synchronization scheme for multiuser OFDM," IEEE J. Sel. Areas Commun., vol. 17, no. 11, pp. 1900-1914, Nov. 1999.

[3] M. Morelli, "Timing and frequency synchronization for the uplink of an OFDMA system," IEEE Trans. Commun., vol. 52, no. 2, pp. 296-306, Feb. 2004.

[4] Z. Cao, U. Tureli, and Y. D. Yao, "Deterministic multiuser carrierfrequency offset estimation for interleaved OFDMA uplink," IEEE Trans. Commun., vol. 52, no. 9, pp. 1585-1594, Sep. 2004.

[5] Z. Cao, U. Tureli, Y. D. Yao, and P. Honan, "Frequency synchronization for generalized OFDMA uplink," in Proc. Globecom, Dec. 2004, pp. 1071-1075.

[6] M. O. Pun, M. Morelli, and C. C. Jay Kuo, "Joint synchronization and channel estimation in uplink OFDMA systems," in Proc. ICASSP, Philadelphia, PA, Mar. 2005, pp. 857-860.

[7] S. Sezginer and P. Bianchi, "Joint frequency offset and channel estimation in the OFDMA uplink: Cramer-Rao bound and training sequence design," in Proc. SPAWC, New York, Jun. 2005, pp. 585-589.

[8] J. J. van de Beek, M. Sandell, and P. O. Borjesson, "ML estimation of timing and frequency offset in OFDM systems," IEEE Trans. Signal. Process., vol. 45, no. 7, pp. 1800-1805, Jul. 1997.

[9] X. Wang, T. T. Tjhung, Y. Wu, and B. Caron, "SER performance evaluation and optimization of OFDM system with residual frequency and timing offsets form imperfect synchronization," IEEE Trans. Broadcast., vol. 49, no. 2, pp. 170-177, Jun. 2003.

[10] H. Lui and U. Tureli, "A high efficient carrier estimator for OFDM communications," IEEE Commun. Lett., vol. 2, no. 4, pp. 104-106, Apr. 1998.

[11] S. Attallah, "Blind estimation of residual carrier offset in OFDM systems," IEEE Signal Process. Lett., vol. 11, no. 2, pp. 216-219, Feb. 2004.

[12] J. Lee and S. Kim, "Residual frequency offset compensation using the approximate SAGE algorithm for OFDM system," IEEE Trans. Commun., vol. 54, no. 5, pp. 765-769, May 2006.

[13] J. A. Fessler and A. O. Hero, "Space-alternating generalized expectation-maximization algorithm," IEEE Trans. Signal Process., vol. 42, no. 10, pp. 2664-2677, Oct. 1994.

[14] B. H. Fleury, M. Tschudin, R. Heddergott, D. Dahlhaus, and K. I. Pedersen, "Channel parameter estimation in mobile radio environments using SAGE algorithm," IEEE J. Sel. Areas Commun., vol. 17, no. 3, pp. 434-450, Mar. 1999.

[15] M. S. El-Tanany, Y. Wu, and L. Hazy, "OFDM uplink for interactive broadband wireless: Analysis and simulation in the presence of carrier, clock and timing errors," IEEE Trans. Broadcast., vol. 49, no. 1, pp. 319, Mar. 2001.

[16] A. P. Dempster, N. M. Laird, and D. B. Rubin, "Maximum-likelihood from the incomplete data via the EM algorithm," J. R. Stat. Soc., vol. 39, no. 1 , pp. $1-17,1977$

[17] Y. Xie and C. N. Georghiades, "Two EM-type channel estimation algorithms for OFDM with transmitter diversity," IEEE Trans. Commun., vol. 51, no. 1, pp. 106-115, Jan. 2003.

[18] D. Huang and K. B. Letaief, "An interference-cancellation scheme for carrier frequency offsets correction in OFDMA systems," IEEE Trans. Commun., vol. 53, no. 7, pp. 1155-1165, Jul. 2005.

[19] P. H. Moose, "A technique for orthogonal frequency division multiplexing frequency offset correction," IEEE Trans. Commun., vol. 42, no. 10, pp. 2908-2914, Oct. 1994.

[20] W. C. Y. Lee, Mobile Communications Engineering. New York: McGraw-Hill, 1982. 